\title{
Scientific Note/Comunicação Cienthica Ocorrência de Aethalion reticulatum Linnaeus (Hemiptera: Aethalionidae) em Tectona grandis Linn. f. (Verbenaceae) em Minas Gerais, Brasil
}

\author{
Thiago Assis Tavares, Adriano Geraldo Fonseca, Fernanda Freitas Sousa ${ }^{\bowtie}$ \& Sebastião Lourenço de Assis Júnior
}

Universidade Federal dos Vales do Jequitinhonha e Mucuri

\section{EntomoBrasilis 11 (3): 220-222 (2018)}

Resumo. A Teca (Tectona grandis Linn. F.) é uma espécie arbórea oriunda do sudeste da Ásia que foi introduzida no Brasil nos anos 6o. Atualmente seu cultivo está em franca expansão em vários estados. Apesar desta espécie apresentar relativa resistência, alguns insetos têm sido reportados em povoamentos homogêneos, com possibilidades de provocar perdas em sua produção. Foi observada a ocorrência de uma cigarrinha em teca no município de Paulistas, Minas Gerais, Brasil. A espécie Aethalion reticulatum Linnaeus (Hemiptera: Aethalionidae) foi encontrada em galhos e folhas de árvores com três anos de idade. A ocorrência dessa cigarrinha indica a colonização e adaptação por insetos nativos, que futuramente poderão alcançar o status de praga da cultura da teca.

Palavras chave: Cigarrinhas; Colonização; Honeydew; Succívoros; Teca.

Occurrence of Aethalion reticulatum Linnaeus (Hemiptera: Aethalionidae) in Tectona grandis Linn. f. (Verbenaceae) in Minas Gerais, Brazil

\begin{abstract}
Teca (Tectona grandis Linn. F.) is a tree species originating in Southeast Asia wich was introduced in Brazil in the 1960s. Currently, its cultivation is booming in several states. Although this species shows relative resistance, some insects have been reported in homogenous stands, with possibilities of causing losses in their production. It was observed the occurrence of a spittlebug in teak in the municipality of Paulistas, Minas Gerais, Brazil. The specie Aethalion reticulatum Linnaeus (Hemiptera: Aethalionidae) was found on branches and leaves of three years old trees. The occurrence of this spittlebug indicates colonization and adaptation by native insects, which may eventually reach the pest status of teak culture.
\end{abstract}

Keywords: Colonization; Honeydew; Spittlebug; Succivorous; Teak.

$\sqrt{L}$ Tectona grandis Linn. F., conhecida como teca pertence à família Verbenaceae e é nativa das florestas tropicais do Sudeste Asiático. É uma árvore caducifólia de grande porte e tronco normalmente retilíneo. Suas propriedades permitem múltiplas aplicações como móveis, pisos, decoração e construção naval (MACEDo et al. 2005).

A teca é cultivada desde o século XVIII. No entanto, só foi introduzida no Brasil no final da década de 1960, sendo o estado do Mato Grosso o de maior área plantada (Oliveira et al. 2014). Plantios dessa cultura têm se destacado na região Amazônica, mas também têm sido promissores em outros estados, especialmente Minas Gerais (MACEDo et al. 2005). Atualmente, a área plantada com essa cultura, no Brasil, ultrapassa 87 mil ha (IBÁ 2016). No entanto, os plantios são, ainda, insuficientes para suprir a demanda por esta madeira (FIgUEIREDo et al. 2005).

Fatores abióticos como o estresse hídrico podem comprometer o desenvolvimento da teca (Lechinoski et al. 2007). Mas, a influência de fatores bióticos é mais conhecida, e dentre estes destacam-se os nematoides (KULKARNi et al. 2011) e os insetos, que podem afetar a cultura desde a fase de mudas.

Os povoamentos homogêneos proporcionam condições favoráveis para a ocorrência de insetos-praga, devido à simplificação do ecossistema, mudanças ambientais, oferta excessiva de alimento e menor eficiência de inimigos naturais. Estes fatores contribuem para o aumento das populações desses organismos que podem causar prejuízos à cultura (SiLva et al. 2013).

Doze artrópodes foram encontrados associados à esta planta na Índia (PANDEY et al. 2010), e o inseto mais conhecido nesta cultura é a Eutectona macheralis (Walker) (Lepidoptera: Pyralidae) (РАтнак et al. 2014). No Brasil, por ser exótica, ainda é pequena a entomofauna associada à teca, sendo raros os relatos de organismos causando danos à essa cultura. Os principais insetos associados à teca no Brasil são a lagarta-da-
Edited by:

William Costa Rodrigues

Article History:

Received: 11.xi.2017

Accepted: $10 . v .2018$
Corresponding author:

Fernanda Freitas Sousa

Bf fernandafreitas81@gmail.com

(3) https://orcid.org/0000-0002-6763-7837
Funding agencies:

$\leftrightarrow$ Without funding declared 
teca Hyblaea puera Cramer (Lepidoptera: Hyblaeidae) (PerEs FilHo et al. 2002), Sinoxylon conigerum Gerstäcker (Coleoptera: Bostrichidae) (Peres Filho et al. 2006) e Cornitermes snyderi Emerson (Isoptera: Termitidae) (PEREs FilHo et al. 2012). Além destes, também, já foram encontrados nessa cultura: cupins de solo, formigas cortadeiras, grilos, lagartas e coleópteros (PAEs et al. 2014; Silva et al. 2013).

Insetos nativos, com o passar do tempo, podem se adaptar às plantas introduzidas e vir a causar danos, com prejuízos consideráveis. Deste modo, objetivou-se relatar a ocorrência de uma cigarrinha na teca em Minas Gerais.

A ocorrência das cigarrinhas na teca foi observada em setembro de 2016 em visita periódica a um povoamento florestal de plantas de $T$. grandis localizado no município de Paulistas, no estado de Minas Gerais ( $\left.18^{\circ} 22^{\prime} 20.07^{\prime \prime} \mathrm{S}, 4^{\circ}{ }^{\circ} 49^{\prime} 4.76^{\prime \prime} \mathrm{O}\right)$. O clima nessa localidade, segundo classificação de Köppen, é Cwa - temperado úmido com inverno seco e verão quente. A altitude média é 690 $\mathrm{m}$, sendo a temperatura e precipitação média anual de $20^{\circ} \mathrm{C} \mathrm{e}$ $1.180 \mathrm{~mm}$, respectivamente.

Na ocasião da observação as árvores estavam com três anos e possuíam altura média de 3 metros. Essas plantas encontravamse em fase vegetativa quando foram observados imaturos e adultos de cigarrinha sugando a seiva no pecíolo foliar e região apical. Foram tiradas fotografias do inseto no local e realizada coleta manual de alguns exemplares. Estes foram acondicionados em potes contendo álcool a 70\% e enviados ao Laboratório de Entomologia Florestal da Universidade Federal dos Vales do Jequitinhonha e Mucuri - UFVJM para identificação.

Os indivíduos foram identificados como Aethalion reticulatum Linnaeus (Hemiptera: Aethalionidae) (Figura 1).

A espécie $A$. reticulatum é conhecida popularmente como cigarrinha do pedúnculo ou das fruteiras e pertencem à superfamília Membracoidea. Mede cerca de $10 \mathrm{~mm}$ de comprimento e apresenta cor marrom ferrugínea com nervuras das asas esverdeadas e salientes. Ovipositam aproximadamente 100 ovos envoltos por uma substância de cor acinzentada nos galhos ou pedúnculos das plantas. Apresentam cuidado parental, tanto para com ovos quanto para as ninfas. Seu ciclo de vida é de 110 dias, podendo apresentar três gerações anuais (GALLo et al. 2002).

Em espécies agrícolas os danos causados por insetos sugadores ocorrem em praticamente todos os estádios fenológicos das culturas. Em estágios iniciais pode causar a morte de plântulas, reduzindo a densidade populacional, ocasionando perdas na produtividade. $\mathrm{O}$ atraso no crescimento das plantas é devido às cigarrinhas se alimentarem por meio da sucção da seiva diretamente do floema, podendo provocar dano direto. Além disso, podem também causar danos indiretos devido a transmissão de viroses e injeção de compostos tóxicos pela saliva durante a alimentação.

Picadas sucessivas e extração da seiva por cigarrinhas podem causar deformações e encarquilhamento de folhas, morte de brotos terminais, perda de dominância apical e superbrotamento de galhos laterais. Altas infestações da cigarrinha A. reticulatum podem levar a depleção de nutrientes, irregularidades no crescimento e consequente desuniformidade em plantios de eucalipto (MenEzes et al. 2012).

Por ser generalista, A. reticulatum apresenta potencial nocivo às novas implantações de reflorestamento com teca devido a absorção de nutrientes necessários ao desenvolvimento da planta e pelo consequente encarquilhamento das folhas.

A. reticulatum foi observada pela primeira vez predando $T$. grandis. A predação por este inseto pode causar danos diretos e indiretos nessa importante cultura, podendo ocasionar perdas econômicas e alterar seu status de praga para essa cultura.

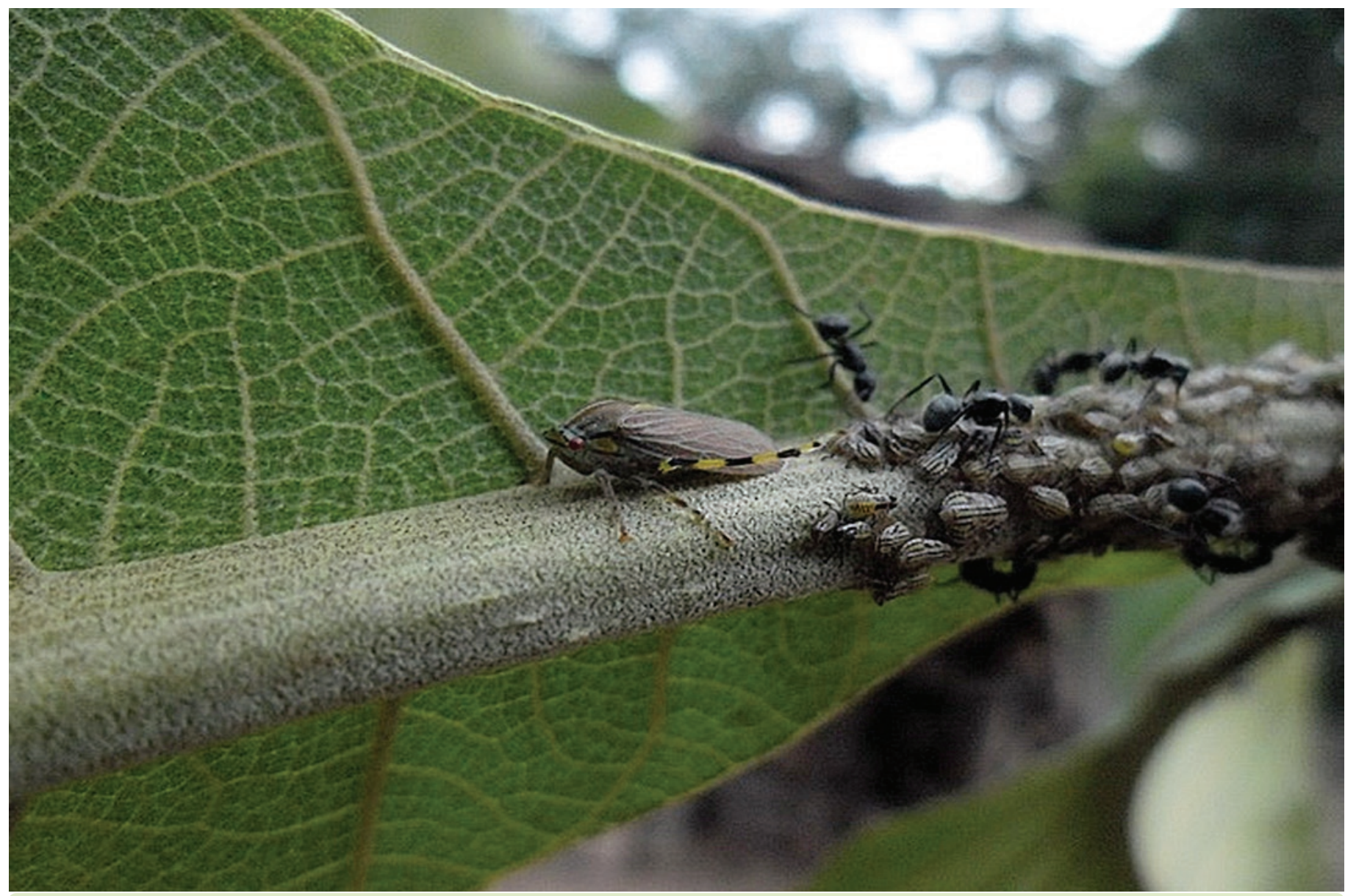

Figura 1: Aethalion reticulatum Linnaeus (Hemiptera: Aethalionidae) atacando Tectona grandis Linn. f. no município de Paulistas-MG. 


\section{REFERÊNCIAS}

Figueiredo, E.O., A.D. Oliveira \& J.R.S. Scolforo, 2005. Análise econômica de povoamentos não desbastados de Tectona grandis L.f., na microrregião do baixo Rio Acre. Cerne, 11: 342-353.

Gallo, D., O. Nakano, S. Silveira Neto, R.P.L. Carvalho, G.C. Batista, E. Berti Filho, J.R.P. Parra, R.A. Zucchi, S.B. Alves, J. Vendramin, L.C. Marchini, J.R.S. Lopes \& C. Omoto. 2002. Manual de Entomologia Agrícola. Piracicaba, FEALQ, $920 \mathrm{p}$.

IBÁ (Indústria Brasileira de Árvores), 2016. Anuário Estatístico da Indústria Brasileira de Árvores: ano base 2015. Brasília, DF:100 p.

Kulkarni, N., S. Paunikar \& S.S. Hussaini, 2011. Susceptibility of Teak Skeletonizer, Eutectona machaeralis (Walker) to the EPN, Heterorhabditis indica Poinar. World Journal of Zoology, 6: 33-39.

Lechinoski, A., J.M.N. Freitas, D.S. Castro, A.K.S. Lobato, C.F. Oliveira Neto \& R.L.M. Cunha, 2007. Influência do estresse hídrico nos teores de proteínas e aminoácidos solúveis totais em folhas de teca (Tectona grandis L. f.). Revista Brasileira de Biociências, 5: 927-929.

Macedo, R.L.G., J.E. Gomes, N. Venturin \& B.G.V. Salgado, 2005. Desenvolvimento inicial de Tectona grandis Lf (Teca) em diferentes espaçamentos no município de Paracatu, MG. Cerne, 11(1): 61-69.

Menezes, C.W.G., M.A. Soares, S.L. Assis Júnior, A.J. Fonseca, E.M. Pires \& J. Barbosa dos Santos, 2012. Novos insetos sugadores (Hemiptera) atacando Eucalyptus cloeziana (Myrtaceae) em Minas Gerais, Brasil. EntomoBrasilis, 5(3): 246-248.

Oliveira, R.G.M.G., E. Berti Filho, O. Peres Filho, F.P. Sales \& J.A.C. Cunha, 2014. Diversity of hymenopteran parasitoids (Hymenoptera: Chalcididae) associated with teak (Tectona grandis) forests. Advances in Forestry Science, 1: 59-64. Disponível em <http://www.periodicoscientificos.ufmt.br/ ojs/index.php/afor/article/view/1736>.
Paes, J.B., P.L. Loiola, W.A. Capelini , L.L. Santos \& H.J.G. Santos Junior, 2014. Entomofauna associada a povoamentos de teca localizados no sul do Estado do Espírito Santo. Pesquisa Florestal Brasileira, 34: 125-132. DOI: https://doi.org/10.4336/2014.pfb.34.78.675.

Pandey, V., A.K. Sing \& R.P. Sharma, 2010. Biodiversity of insect pests associated with teak (Tectona grandis Lf) in Eastern Uttar Pradesh of India. Research Journal of Forestry, 4: 136144. DOI: https://doi.org/10.3923/rjf.2010.136.144.

Pathak, H., S. Maru \& S.C. Silawat, 2014.Field study for the effectiveness of some plants leaf extracts against insect Eutectona macheralis or Teak skeletonizer in forest nursery of Indore, Madhya Pradesh. American Journal of Agriculture and Forestry, 2: 110-113. DOI: https://doi.org/10.11648/j. ajaf.20140204.12.

Peres Filho, O., A. Dorval \& E. Berti Filho, 2002. Ocorrência de Hyblaea puera (Cramer, 1777) (Lepidoptera: Hyblaeidae) em Teca no Brasil. Bragantia, 61: 59-60. DOI: https://doi.org/10.1590/s0006-87052002000100009.

Peres Filho, O., E.P. Teixeira, M.L.M. Bezerra, A. Dorval \& E. Berti Filho, 2006. First record of Sinoxylon conigerum Gerstäcker (Coleoptera: Bostrichidae) in Brazil. Neotropical Entomology, 35: 712-713. DOI: https://doi.org/10.1590/ s1519-566x2006000500023.

Peres Filho, O., J.C. Souza, M.D. Souza \& A. Dorval, 2012. Distribuição espacial de cupinzeiros de Cornitermes snyderi (Isoptera: Termitidae) e sua associação com teca. Pesquisa Florestal Brasileira, 32: 175-181. DOI: https://doi.org/10.4336/2012.pfb.32.70.59.

Silva, C.V.M., K.Y. Yamaki \& A.G. Silva, 2013. Identificação e caracterização da entomofauna em plantios de teca (Tectona grandis). Nucleus, 10: 207-218. DOI: https://doi.org/10.3738/1982.2278.879.

\section{Suggestion citation:}

Tavares, T.A., A.G. Fonseca, F.F. Sousa \& S.L. de Assis Júnior, 2018. Ocorrência de Aethalion reticulatum Linnaeus (Hemiptera: Aethalionidae) em Tectona grandis Linn. f. (Verbenaceae) em Minas Gerais, Brasil. EntomoBrasilis, 11 (3): 220-222.

Available on: $\underline{\text { doi:10.12741/ebrasilis.v11i3.752 }}$
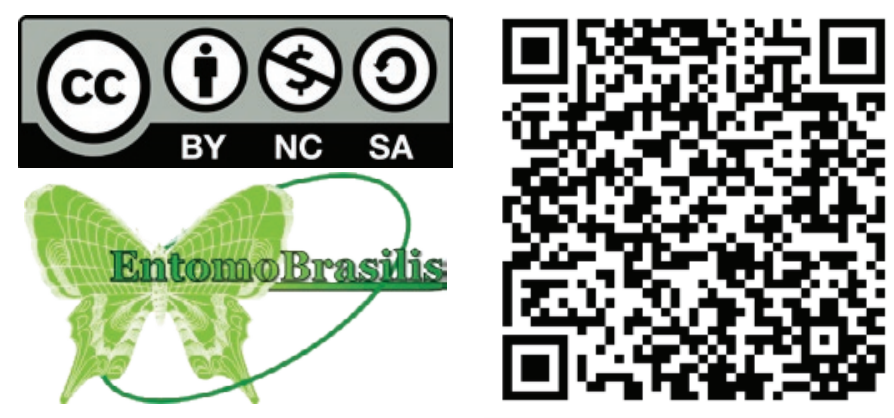